\title{
Medium-term impact of COVID-19 on pulmonary function, functional capacity and quality of life
}

\author{
Fabio Anastasio $\mathbb{1}^{1}$, Sarah Barbuto ${ }^{1}$, Elisa Scarnecchia ${ }^{1,2}$, Paolo Cosma ${ }^{1}$, Alessandro Fugagnoli ${ }^{1}$, \\ Giulio Rossi ${ }^{1}$, Mirco Parravicini ${ }^{1}$ and Pierpaolo Parravicini ${ }^{1}$
}

${ }^{1}$ ASST Valtellina and Alto Lario, Eugenio Morelli Hospital, Sondalo, Italy. ${ }^{2}$ Medical and Surgical Sciences Dept, Alma Mater Studiorum, Bologna University, Bologna, Italy.

Corresponding author: Fabio Anastasio (fabio.anastasio@asst-val.it)

Shareable abstract (@ERSpublications)

COVID-19 severe lung involvement can reduce respiratory performance in the medium- to longterm. Respiratory rehabilitation is recommended in COVID-19 survivors showing severe clinical and radiological signs of disease. https://bit.ly/3jCxNOs

Cite this article as: Anastasio F, Barbuto S, Scarnecchia E, et al. Medium-term impact of COVID-19 on pulmonary function, functional capacity and quality of life. Eur Respir J 2021; 58: 2004015 [DOI: 10.1183/13993003.04015-2020].

Copyright @The authors 2021

This version is distributed under the terms of the Creative Commons Attribution Non-Commercial Licence 4.0. For commercial reproduction rights and permissions contact permissions@ersnet.org

Received: 31 Oct 2020 Accepted: 3 Feb 2021

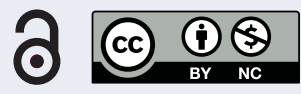

\section{Abstract}

Background Coronavirus disease 2019 (COVID-19) has spread worldwide, having a dramatic impact on healthcare systems. The aim of this study is to evaluate mid-term clinical impact of COVID-19 on respiratory function.

Methods 379 patients were evaluated 4 months after severe acute respiratory syndrome coronavirus 2 (SARS-CoV-2) diagnosis. Patients were divided in two groups based on the presence of pneumonia during COVID-19. Clinical conditions, quality of life, symptomatology, 6-min walk test, pulmonary function test with spirometry and diffusing capacity of the lung for carbon monoxide were analysed. Data were compared to clinical evolution during COVID-19 (development of acute respiratory distress syndrome, need of invasive mechanical ventilation, partial oxygen saturation $\left(S_{\mathrm{pO}_{2}}\right)$ /inspiratory oxygen fraction $\left(F_{\mathrm{IO}_{2}}\right)$ ratio and pneumonia severity index (PSI)).

Results After a median 135 days, 260 (68.6\%) out of 379 patients referred at least one symptom. Patients who developed pneumonia during COVID-19 showed lower $S_{\mathrm{pO}_{2}}$ at rest $(\mathrm{p}<0.001), S_{\mathrm{pO}_{2}}$ during 6-min walk test $(\mathrm{p}<0.001)$, total lung capacity $(\mathrm{p}<0.001)$, airway occlusion pressure after $0.1 \mathrm{~s}\left(P_{0.1}\right)(\mathrm{p}=0.02)$, $P_{0.1} /$ maximal inspiratory pressure ratio $(\mathrm{p}=0.005)$ and higher Borg category-ratio scale $(\mathrm{p}=0.006)$ and modified Medical Research Council breathlessness scale $(\mathrm{p}=0.003)$, compared to patients without pneumonia. $S_{\mathrm{pO}_{2}} / F_{\mathrm{IO}_{2}}$ ratio and PSI during SARS-CoV-2 pneumonia were directly associated with midterm alteration of $S_{\mathrm{pO}_{2}}$ at rest $(\mathrm{p}<0.001)$ and during 6-min walk test $(\mathrm{p}<0.001)$, residual volume $(\mathrm{p}<0.001)$, total lung capacity $(\mathrm{p}<0.001$ and $\mathrm{p}=0.003$, respectively) and forced vital capacity $(\mathrm{p}=0.004$ and $\mathrm{p}=0.03$, respectively).

Conclusion Lung damage during COVID-19 correlates to the reduction of pulmonary function 4 months after acute infection.

\section{Introduction}

The respiratory system is subjected to major involvement during coronavirus disease 2019 (COVID-19), due additionally to the hyperactive host immune response and inflammatory organ injury, but there is no evidence about organ dysfunction in the mid- and long-term. From previous experience with coronavirus lung involvement of severe acute respiratory syndrome (SARS) and Middle-East respiratory syndrome (MERS), radiological abnormalities, impairment of pulmonary function and reduced exercise capacity improve over time, but may persist in some for months or even years [1-3]. Evidence about pulmonary function tests after discharge among COVID-19 patients is currently limited to few retrospective studies with small samples showing, in severe COVID-19, a reduction of forced vital capacity (FVC), diffusing capacity (transfer factor) of the lung for carbon monoxide $\left(D_{\mathrm{LCO}}\right)$, total lung capacity (TLC), 6-min walk 
distance (6MWD) and impairment in respiratory muscle strength with need for respiratory rehabilitation [4-7].

The aim of our study (Cardio-Respiratory Sequelae of COVID-19) is to evaluate respiratory function 4 months after diagnosis in patients who survive severe acute respiratory syndrome coronavirus 2 (SARS-CoV-2) infection and the difference between patients with or without initial lung involvement. The second outpatient visit will be completed 12 months after diagnosis.

\section{Methods}

\section{Study design}

Patients aged between 18 and 80 years with COVID-19 diagnosis were recruited. Patients were evaluated at the time of diagnosis and then after a median of 4 months for respiratory function during an outpatient visit. The inclusion criterion was COVID-19 diagnosis by PCR on pharyngeal swab or on alveolarbronchial washing in cases of double-negative swab. The exclusion criterion was previous diagnosis of pulmonary disease, excluding asthma. Data on COVID-19 evolution were collected retrospectively; development of acute respiratory distress syndrome (ARDS), need of invasive mechanical ventilation (IMV), partial oxygen saturation $\left(\mathrm{S}_{\mathrm{pO}_{2}}\right)$ /inspiratory oxygen fraction $\left(F_{\mathrm{IO}_{2}}\right)$ ratio, pneumonia severity index (PSI), steroid therapy, pulmonary embolism and worst blood tests (C-reactive protein, procalcitonin, ferritin, d-dimer, high-sensitivity troponin I, neutrophil/lymphocyte ratio and lactate dehydrogenase) were acquired at the time of diagnosis.

ARDS was diagnosed according to the Berlin definition: timing of worsening of the respiratory symptoms, diffuse bilateral pneumonia at chest imaging and arterial oxygen tension $/ F_{\mathrm{IO}_{2}} \leqslant 300 \mathrm{mmHg}$ with positive end-expiratory pressure $\geqslant 5 \mathrm{cmH}_{2} \mathrm{O}$ [8]. Patients with ARDS well-controlled by noninvasive ventilation or high-flow nasal oxygen were not admitted to the intensive care unit (ICU) due to the lack of beds [9]. The use of noninvasive ventilation or high-flow nasal oxygen were not evaluated due to the heterogeneity of treatments and lack of full data.

Patient history, body mass index (BMI), smoking habits, vital signs (respiratory rate, $S_{\mathrm{pO}_{2}}$, heart rate, systolic blood pressure, diastolic blood pressure), symptoms and therapy were collected. In addition, the impact of exertional breathlessness during the prior 2 weeks was measured using the modified Medical Research Council (mMRC) breathlessness scale. Patients were subjected to pulmonary function tests, 6-min walk test (6MWT) and self-reported health variables.

\section{Pulmonary function tests}

Outpatient pulmonary function tests were performed using MasterScreen Body (Jaeger, Wurzburg, Germany) and were performed by technicians at the pulmonary function laboratory. Forced expiratory volume in $1 \mathrm{~s}\left(\mathrm{FEV}_{1}\right), \mathrm{FVC}, \mathrm{FEV}_{1} / \mathrm{FVC}$ ratio, TLC, residual volume (RV), RV/TLC, $D_{\mathrm{LCO}}$ /alveolar volume $\left(V_{\mathrm{A}}\right)$, maximal voluntary ventilation $(\mathrm{MVV})$, maximal inspiratory pressure (MIP), maximal expiratory pressure (MEP), the airway occlusion pressure $0.1 \mathrm{~s}$ after the beginning of inspiration $\left(P_{0.1}\right)$, the airway occlusion pressure in relation to maximal inspiratory drive $\left(P_{0.1} / \mathrm{MIP}\right)$, specific airway resistance $\left(R_{\mathrm{aw}}\right)$, peak expiratory flow (PEF) and maximum expiratory flows at $75 \%, 50 \%$ and $25 \%$ of $\mathrm{FVC}$ $\left(\mathrm{MEF}_{75 \%}, \mathrm{MEF}_{50 \%}\right.$ and $\mathrm{MEF}_{25 \%}$, respectively) were included in the analysis. For each patient, parameters were expressed as percentage of a theoretical value calculated by Global Lung Function 2012 equations [10].

\section{MWT}

The 6MWT was performed on room air under the supervision of a respiratory therapists in the pulmonary rehabilitation department according to American Thoracic Society guidelines [11]. BlueNight Oximeter (Sleepinnov Technology SAS, Moirans, France) pulse oximeter was used for recordings. Borg category-ratio scale (CR10) and self-reported intensity of exertion on the Borg rating of perceived exertion were collected before and after 6MWT.

\section{Per-group analysis}

Patients were divided in two groups according to the presence of radiological signs of pneumonia during acute COVID-19 to assess the difference of pulmonary function, 6MWD and health variables at mid-term. Therefore, variables were analysed by the presence/absence of ARDS, need of IMV, $S_{\mathrm{PO}_{2}} / F_{\mathrm{IO}_{2}}$ ratio and PSI. 
Health-related quality-of-life survey and International Physical Activity Questionnaire

At the health check, participants were requested to self-complete an Italian version of the 12-item Short Form survey (SF-12) and International Physical Activity Questionnaire (IPAQ). The SF-12 includes eight subscales: physical functioning, role (physical), bodily pain, general health, vitality, social functioning, role (emotional) and mental health. These were summarised into two scales: a physical component score (PCS) and a mental component score (MCS), in accordance with the guidelines for the SF-12 instrument [12]. Both scores ranged between 0 and 100, with a higher score indicating better health.

To evaluate the level of physical activity, the IPAQ was used. Patients answered questions regarding activities undertaken during a week at work and at home, means of transport and leisure. Patients were then classified according to the level of physical activity. In the high-intensity group, those who practiced vigorous activity $\geqslant 3$ days per week (or combinations equivalent to 3000 metabolic equivalents (METs) per week) were considered; moderate intensity described $\geqslant 3$ days per week of vigorous intensity activity for $\geqslant 20$ min (or combinations equivalent to 600 METs per week); and the low-intensity group included those who did not correspond to any of the other categories cited [13].

\section{Statistical analysis}

Continuous variables are expressed as median (interquartile range (IQR)) and the categorical variables are presented as n (\%). Differences between groups were assessed by median test and Kruskal-Wallis test. Chi-squared statistics were used to assess differences between categorical variables. Pearson's correlation coefficient and Cox regression were used to study relations between variables. Statistical analysis was performed using SPSS (version 26; SPSS, Chicago, IL, USA) and a p-value of $\leqslant 0.05$ was considered statistically significant.

\section{Ethical approval}

Ethical approval for the Cardio-Respiratory Sequelae of COVID-19 study was granted by the ethics committee of Brianza (Italy) on 6 August 2020. Written informed consent was obtained from all subjects.

\section{Results}

\section{General characteristics}

From 1 March 2020 to 1 June 2020, 1464 cases of SARS-CoV-2 positivity were detected at our hospital. 594 patients were hospitalised; 64 of them were admitted to the ICU and supported by IMV. Among the total sample, 150 patients died. A total of 379 random selected patients were evaluated after a median (IQR) 135 (102-175) days after the onset of symptoms of COVID-19. The age range was 20-80 years, median (IQR) 56 (49-63) years. 174 (45.9\%) patients were male. Median (IQR) BMI was 25.2 (22.6-28.7) $\mathrm{kg} \cdot \mathrm{m}^{-2}$. Pre-existing comorbidities are reported in table 1.25 (6.6\%) patients were active smokers and 128 (33.8\%) patients were ex-smokers, At the outpatient clinical follow-up, 211 (69.9\%) patients referred persistent COVID-19 symptoms at the time of this visit. Exertional dyspnoea (42.7\%), weakness (29.8\%), joint and muscular pain (13.7\%), thoracic pain (11.9\%), anosmia and ageusia (10.3\%) and depression (8.2\%) were the most reported symptoms at evaluation (table 1). 60 (15.8\%) patients had an mMRC score $\geqslant 2$. A clear difference was found between referred dyspnoea and mMRC.

\section{COVID-19 severity}

Among 379 evaluated patients, 222 had developed pneumonia. Among these 222 patients, 143 (64.4\%) were hospitalised at our COVID department and 135 (60.8\%) required oxygen supplementation. ARDS occurred in 61 (27.5\%) patients and 34 (15.3\%) of them were admitted to ICU for IMV. Seven (3.2\%) patients presented pulmonary embolism during acute COVID. During hospitalisation, steroid was administered in 42 (18.9\%) patients with pneumonia and eight (5.1\%) patients without pneumonia. Table 2 shows laboratory and clinical parameters of pneumonia patients.

\section{Pneumonia versus non-pneumonia analysis}

Differences in general characteristics between patients with pneumonia during COVID-19 and patients without it are shown in table 1. Patients with respiratory failure during acute COVID-19 were older $(p<0.001)$, male $(p<0.001)$, with higher BMI $(p<0.001)$, and had a greater prevalence of obesity $(\mathrm{p}<0.001)$, hypertension $(\mathrm{p}<0.001)$, CVD $(\mathrm{p}=0.007)$, diabetes $(\mathrm{p}=0.003)$, CKD $(\mathrm{p}=0.01)$ and current smoking $(\mathrm{p}<0.001)$.

Regarding mid-term vital parameters, respiratory tests and 6MWT (table 3), patients who developed pneumonia during COVID-19 showed higher systolic $(\mathrm{p}=0.002)$ and diastolic blood pressure $(\mathrm{p}=0.002)$, CR10 ( $\mathrm{p}=0.006)$, mMRC ( $\mathrm{p}=0.003)$, CR10 after 6MWT $(\mathrm{p}=0.04)$, PEF $(\mathrm{p}=0.009)$ and $\mathrm{MEF}_{75}(\mathrm{p}=0.02)$ and lower $S_{\mathrm{pO}_{2}}$ at rest $(\mathrm{p}<0.001), S_{\mathrm{pO}_{2}}$ during 6MWT $(\mathrm{p}<0.001), P_{0.1}(\mathrm{p}=0.02), P_{0.1} / \mathrm{MIP}(\mathrm{p}=0.005)$ and 
TABLE 1 Characteristics of patients and difference between patients with pneumonia during coronavirus disease 2019 and patients without it

\begin{tabular}{|c|c|c|c|c|c|c|c|c|c|c|}
\hline & \multicolumn{4}{|c|}{ All patients } & \multicolumn{6}{|c|}{ Pneumonia } \\
\hline & Total & Not pneumonia & Pneumonia & $\mathrm{p}$-value & Not ARDS & ARDS & $\mathrm{p}$-value & Not IMV & IMV & $\mathrm{p}$-value \\
\hline Patients & 379 & 157 & 222 & & 161 & 61 & & 188 & 34 & \\
\hline Age years & $56(49-63)$ & $52(39-58)$ & $58(53-67)$ & $<0.001$ & $58(52-64)$ & $66(58-73)$ & $<0.001$ & $58(52-66)$ & $60(57-72)$ & 0.05 \\
\hline Male & $174(45.9)$ & $47(29.9)$ & $127(57.2)$ & $<0.001$ & $79(47.6)$ & $54(83.1)$ & $<0.001$ & $96(51.1)$ & $31(91.2)$ & $<0.001$ \\
\hline $\mathrm{BMI} \mathrm{kg} \cdot \mathrm{m}^{-2}$ & $25.2(22.6-28.7)$ & $23.9(21.5-27.8)$ & $26.2(23.4-29.1)$ & $<0.001$ & $25.3(23.0-29.3)$ & $27.1(24.8-28.7)$ & 0.18 & $25.9(23.4-29.3)$ & $26.4(23.8-28.8)$ & 0.94 \\
\hline \multicolumn{11}{|l|}{ Comorbidities } \\
\hline Hypertension & $112(29.6)$ & $24(15.3)$ & $88(39.6)$ & $<0.001$ & $56(33.7)$ & $36(55.4)$ & 0.003 & $68(36.2)$ & $20(58.8)$ & 0.01 \\
\hline CVD $^{\#}$ & 44 (11.6) & $10(6.4)$ & $34(15.3)$ & 0.007 & $22(13.3)$ & $14(21.5)$ & 0.12 & $29(15.4)$ & $5(14.7)$ & 0.91 \\
\hline Diabetes mellitus & $24(6.3)$ & $3(1.9)$ & $21(9.5)$ & 0.003 & $12(7.2)$ & $9(13.8)$ & 0.12 & $17(9.0)$ & $4(11.8)$ & 0.62 \\
\hline Neoplasia & $19(5.0)$ & $8(5.1)$ & $11(5.0)$ & 0.95 & $9(5.4)$ & $4(6.2)$ & 0.83 & $9(4.8)$ & $2(5.9)$ & 0.78 \\
\hline Asthma & $32(8.4)$ & $12(7.6)$ & $20(9.1)$ & 0.64 & $21(12.7)$ & $0(0.0)$ & 0.003 & $20(10.6)$ & $0(0.0)$ & 0.05 \\
\hline Hepatic disease & $5(1.3)$ & $1(0.6)$ & $4(1.8)$ & 0.33 & $2(1.2)$ & $2(3.1)$ & 0.33 & $4(2.1)$ & $0(0.0)$ & 0.39 \\
\hline CKD & $13(3.4)$ & $1(0.6)$ & $12(5.4)$ & 0.01 & $10(6.0)$ & $3(46)$ & 0.68 & $10(5.3)$ & $2(5.4)$ & 0.89 \\
\hline Obesity & 59 (15.6) & $11(7.0)$ & $48(21.6)$ & $<0.001$ & $38(22.9)$ & $11(16.9)$ & 0.32 & $42(22.3)$ & $6(17.6)$ & 0.54 \\
\hline Smoking history & $128(33.8)$ & $49(31.2)$ & 79 (35.6) & 0.38 & $49(29.5)$ & $36(55.4)$ & $<0.001$ & 59 (31.4) & $20(58.8)$ & 0.002 \\
\hline Current smoker & $25(6.6)$ & $20(12.7)$ & $5(2.3)$ & $<0.001$ & $5(3.0)$ & $0(0.0)$ & 0.16 & $5(2.7)$ & $0(0.0)$ & 0.33 \\
\hline Dyspnoea & $162(42.7)$ & $55(35.0)$ & $107(48.2)$ & 0.01 & $81(48.8)$ & $28(43.1)$ & 0.47 & $88(46.8)$ & $19(55.9)$ & 0.27 \\
\hline Weakness & $113(29.8)$ & $54(34.4)$ & $59(26.6)$ & 0.11 & $47(28.3)$ & $13(20.0)$ & 0.21 & $54(28.7)$ & $5(14.7)$ & 0.10 \\
\hline Joint and muscular pain & $52(13.7)$ & $16(10.2)$ & $36(16.2)$ & 0.09 & $24(14.5)$ & $12(18.5)$ & 0.43 & $27(14.4)$ & $9(26.5)$ & 0.07 \\
\hline Thoracic pain & 45 (11.9) & $22(14.0)$ & $23(10.4)$ & 0.29 & $22(13.3)$ & $2(3.1)$ & 0.02 & $22(11.7)$ & $1(2.9)$ & 0.13 \\
\hline Anosmia and ageusia & $39(10.3)$ & $17(10.8)$ & $22(9.9)$ & 0.78 & 19 (11.4) & $3(4.6)$ & 0.11 & $19(10.1)$ & $3(8.8)$ & 0.81 \\
\hline Depression & $31(8.2)$ & $11(7.0)$ & $20(9.0)$ & 0.48 & $17(10.2)$ & $3(4.6)$ & 0.17 & $19(10.1)$ & $1(2.9)$ & 0.18 \\
\hline Cough & $23(6.1)$ & $11(7.0)$ & $12(5.4)$ & 0.53 & $13(7.8)$ & $0(0.0)$ & 0.02 & $12(6.4)$ & $0(0.0)$ & 0.13 \\
\hline Heart palpitations & $23(6.1)$ & $11(7.0)$ & $12(5.4)$ & 0.53 & $7(4.2)$ & $5(7.7)$ & 0.29 & $7(3.7)$ & $5(14.7)$ & 0.01 \\
\hline Headache & $20(5.3)$ & $11(7.0)$ & $9(4.1)$ & 0.21 & $5(3.0)$ & $4(6.2)$ & 0.27 & $6(3.2)$ & $3(8.8)$ & 0.13 \\
\hline Sleeping disorders & $16(4.2)$ & $5(3.2)$ & $11(5.0)$ & 0.39 & $7(4.2)$ & $4(6.2)$ & 0.54 & $7(3.7)$ & $4(11.8)$ & 0.05 \\
\hline Hair loss & $12(3.2)$ & $6(3.8)$ & $6(2.7)$ & 0.55 & $5(3.0)$ & $1(1.5)$ & 0.52 & $5(2.7)$ & $1(2.9)$ & 0.93 \\
\hline Memory disorders & $10(2.6)$ & $4(2.5)$ & $6(2.7)$ & 0.92 & $5(3.0)$ & $1(1.5)$ & 0.52 & $5(2.7)$ & $1(2.9)$ & 0.93 \\
\hline Dizziness & $9(2.4)$ & $1(0.6)$ & $8(3.6)$ & 0.06 & $6(3.2)$ & $2(3.1)$ & 0.83 & $6(3.2)$ & $2(5.9)$ & 0.44 \\
\hline
\end{tabular}

Data are presented as $\mathrm{n}$, median (interquartile range) or $\mathrm{n}(\%)$, unless otherwise stated. ARDS: acute respiratory distress syndrome; IMV: invasive mechanical ventilation; BMI: body mass index;

CVD: cardiovascular disease; CKD: chronic kidney disease. ": including acute coronary syndrome, chronic heart failure and arrhythmias. 
TABLE 2 Hospitalisation data in patients with pneumonia during coronavirus disease 2019

\begin{tabular}{lcccc} 
& Pneumonia & Non-ARDS & ARDS & p-value \\
\hline Patients & 222 & 161 & 61 & \\
Pulmonary embolism & $7(3.2)$ & $3(1.9)$ & $4(6.6)$ & 0.07 \\
\hline Hospitalisation & $143(64.4)$ & $82(50.9)$ & $161(100)$ & $<0.001$ \\
Worst $\mathrm{S}_{\mathrm{pO}_{2}} / F_{\mathrm{IO}_{2}}$ ratio & $303(200-438)$ & $416(281-447)$ & $117(94-188)$ & $<0.001$ \\
PSI & $70(50-95)$ & $59(43-83)$ & $96(75-113)$ & $<0.001$ \\
Steroid therapy & $42(18.9)$ & $28(17.4)$ & $14(23.0)$ & 0.35 \\
CRP & $94(35-184)$ & $68(15-104)$ & $204(133-267)$ & $<0.001$ \\
\hline PCT & $0.09(0.04-0.27)$ & $0.05(0.03-0.12)$ & $0.39(0.13-0.86)$ & $<0.001$ \\
\hline Ferritin & $1062(272-2930)$ & $393(155-1558)$ & $2828(1062-4152)$ & $<0.001$ \\
\hline d-Dimer & $1380(648-5199)$ & $790(490-2251)$ & $5517(1625-18352)$ & $<0.001$ \\
\hline High-sensitivity troponin I & $5(2.3-12.9)$ & $3.8(1.6-7.0)$ & $10.2(4.3-25.0)$ & $<0.001$ \\
\hline N/L ratio & $5.0(3.1-10.7)$ & $3.8(2.5-7.1)$ & $11.2(5.7-18.0)$ & $<0.001$ \\
\hline LDH & $282(230-413)$ & $238(204-322)$ & $411(319-540)$ & $<0.001$ \\
\hline Days since positive swab & $136(102-177)$ & $146(113-180)$ & $127(97-173)$ & 0.50 \\
\hline Days between positive and negative & $45(34-65)$ & $49(36-73)$ & $43(33-61)$ & 0.21 \\
\multicolumn{1}{r}{ swab } & & & & \\
\hline
\end{tabular}

Data are presented as $n, n(\%)$ or median (interquartile range), unless otherwise stated. ARDS: acute respiratory distress syndrome; $S_{\mathrm{pO}_{2}}$ : partial oxygen saturation; $F_{\mathrm{IO}_{2}}$ : inspiratory oxygen fraction; PSI: pneumonia severity index; CRP: C-reactive protein; PCT: procalcitonin; N/L: neutrophil/leukocyte; LDH: lactate dehydrogenase.

TLC $(\mathrm{p}<0.001)$. Paradoxically, patients without pneumonia involvement showed higher $R_{\mathrm{aw}}(\mathrm{p}=0.03), \mathrm{RV}$ $(p<0.001)$ and lower MIP $(p=0.02)$. Repeating the same analysis excluding obese patients, the results did not change.

In the pneumonia group, patients who reported exertional dyspnoea showed reduction of 6MWD (86\% (74-93\%) versus 90\% (79-103\%), $\mathrm{p}=0.05$ ) without any other difference in pulmonary function.

Impact of pneumonia severity during COVID-19

Evaluating pulmonary involvement in patients with pneumonia, patients who developed ARDS showed higher systolic blood pressure $(\mathrm{p}=0.05)$ and diastolic blood pressure $(\mathrm{p}=0.02)$ and lower $S_{\mathrm{pO}_{2}}$ during 6MWT $(p=0.004)$, FVC $(p=0.004)$ and TLC $(p<0.001)$. Interestingly, patients without ARDS showed higher $R_{\mathrm{aw}}(\mathrm{p}<0.001)$, RV $(\mathrm{p}<0.001)$, TLC $(\mathrm{p}<0.001)$ and RV/TLC $(\mathrm{p}=0.05)$.

Patients who required IMV showed lower 6MWD ( $\mathrm{p}=0.006), S_{\mathrm{pO}_{2}}$ during $6 \mathrm{MWT}(\mathrm{p}=0.002), D_{\mathrm{LCO}} / V_{\mathrm{A}}$ $(\mathrm{p}=0.05)$, TLC $(\mathrm{p}<0.001)$ and FVC $(\mathrm{p}=0.004)$, compared to patients with pneumonia who didn't require IMV. $R_{\text {aw }}(\mathrm{p}<0.001), R V(\mathrm{p}<0.001)$ and RV/TLC $(\mathrm{p}=0.05)$ were higher in patients who did not require IMV.

$\mathrm{S}_{\mathrm{pO}_{2}} / F_{\mathrm{IO}_{2}}$ ratio and PSI during SARS-CoV-2 infection were an important expression of lung damage according to radiological findings. Table 4 shows the correlating $S_{\mathrm{PO}_{2}} / F_{\mathrm{IO}_{2}}$ ratio and PSI during SARS-CoV-2 with the principal parameters investigated during the mid-term outpatient visit. Patients with pneumonia who showed a greater reduction of $S_{\mathrm{pO}_{2}} / F_{\mathrm{IO}_{2}}$ ratio during SARS-CoV-2 acute infection exhibited lower $S_{\mathrm{pO}_{2}}$ at rest $(\mathrm{p}<0.001), S_{\mathrm{pO}_{2}}$ during 6MWT $(\mathrm{p}<0.001)$, MEP $(\mathrm{p}=0.01), \mathrm{RV}(\mathrm{p}<0.001)$, TLC $(\mathrm{p}<0.001)$ and FVC $(\mathrm{p}=0.004)$. Correlation between $S_{\mathrm{pO}_{2}} / F_{\mathrm{IO}_{2}}$ ratio and $S_{\mathrm{pO}_{2}}$ at rest $(\mathrm{p}<0.001)$ and during 6MWT $(\mathrm{p}<0.001)$ was higher in non-ARDS patients. In patients with ARDS, $S_{\mathrm{pO}_{2}} / F_{\mathrm{IO}_{2}}$ ratio correlated positively with $S_{\mathrm{pO}_{2}}$ at rest $(\mathrm{p}=0.05)$, mMRC ( $\left.\mathrm{p}=0.04\right), 6 \mathrm{MWD}(\mathrm{p}=0.02), P_{0.1} / \mathrm{MIP}(\mathrm{p}=0.04)$ and $D_{\mathrm{LCO}} / V_{\mathrm{A}}$ $(\mathrm{p}=0.02)$ and inversely with MVV $(\mathrm{p}=0.02)$ (figure 1). Similarly, PSI correlated inversely with $S_{\mathrm{pO}_{2}}$ at rest $(\mathrm{p}<0.001)$ and during 6MWT $(\mathrm{p}<0.001)$, heart rate during 6MWT $(\mathrm{p}<0.001), R V(\mathrm{p}<0.001)$, TLC $(\mathrm{p}=0.003)$ and FVC $(\mathrm{p}=0.03)$.

\section{Effect of steroid therapy during COVID-19}

Selecting patients with pneumonia during COVID-19, steroid therapy was administered to 42 (18.9\%) patients: 14 (23.0\%) with ARDS and $28(17.4 \%)$ without it. Steroid therapy, corrected for $S_{\mathrm{pO}_{2}} / F_{\mathrm{IO}_{2}}$ ratio, PSI, ARDS development or IMV need, was positively correlated with mMRC $(\mathrm{p}=0.05)$ and $P_{0.1} / \mathrm{MIP}$ 


\begin{tabular}{|c|c|c|c|c|c|c|c|c|c|c|}
\hline & Total & Not pneumonia & Pneumonia & $\mathrm{p}$-value & Not ARDS & ARDS & $\mathrm{p}$-value & Not IMV & IMV & $\mathrm{p}$-value \\
\hline Patients & 379 & 157 & 222 & & 161 & 61 & & 188 & 34 & \\
\hline $\mathrm{S}_{\mathrm{pO}_{2}} \%$ & 97 (96-98) & $97(96-98)$ & $96(95-97)$ & $<0.001$ & $96(95-97)$ & $96(95-97)$ & 0.39 & $96(95-97)$ & $96(95-97)$ & 0.38 \\
\hline Respiratory rate breaths $\cdot \mathrm{min}^{-1}$ & $17(14-20)$ & $17(14-20)$ & $18(14-20)$ & 0.23 & $17(14-21)$ & $18(14-20)$ & 0.84 & $17(14-20)$ & $18(14-21)$ & 0.39 \\
\hline Heart rate beats $\cdot \min ^{-1}$ & $73(65-81)$ & $74(65-82)$ & $72(65-80)$ & 0.15 & $72(65-81)$ & $71(65-82)$ & 0.81 & $72(65-80)$ & $81(70-87)$ & 0.08 \\
\hline SBP & $135(120-146)$ & $128(118-142)$ & 137 (123-149) & 0.002 & $135(123-147)$ & $139(126-157)$ & 0.05 & $136(123-148)$ & $138(126-153)$ & 0.98 \\
\hline DBP & $85(76-92)$ & $82(75-90)$ & $86(78-94)$ & 0.002 & 85 (77-92) & $88(80-98)$ & 0.02 & 85 (77-93) & $88(80-98)$ & 0.34 \\
\hline CR10 at rest & $0.3(0.0-1.0)$ & $0.0(0.0-0.5)$ & $0.3(0.0-2.0)$ & 0.006 & $0.3(0.0-2.0)$ & $0.3(0.0-2.0)$ & 0.88 & $0.3(0.0-2.0)$ & $0.3(0.0-2.0)$ & 0.50 \\
\hline RPE at rest & $11(6-12)$ & $9(6-12)$ & $11(8-12)$ & 0.19 & $11(7-12)$ & $10(8-11)$ & 0.77 & $11(8-12)$ & $8(7-12)$ & 0.35 \\
\hline mMRC & $2(1-2)$ & $1(1-2)$ & $2(1-2)$ & 0.003 & $2(1-2)$ & $2(1-2)$ & 0.98 & $2(1-2)$ & $1(1-2)$ & 0.18 \\
\hline mMRC 0-1 & $319(84.2)$ & $143(91.1)$ & $176(79.3)$ & 0.01 & $128(79.5)$ & $49(80.3)$ & 0.97 & $132(70.2)$ & 31 (91.2) & 0.31 \\
\hline $\mathrm{mMRC} \geqslant 2$ & $60(15.8)$ & $14(8.9)$ & $46(20.7)$ & 0.01 & $33(20.5)$ & $12(19.7)$ & 0.97 & $56(29.8)$ & $3(8.8)$ & 0.31 \\
\hline 6MWD m & $520(420-560)$ & $520(460-580)$ & $500(400-560)$ & 0.006 & $500(400-560)$ & $500(390-540)$ & 0.34 & $500(400-560)$ & $400(320-520)$ & 0.04 \\
\hline 6MWD \% & 88 (77-99) & $88(75-100)$ & 87 (77-98) & 0.86 & 87 (77-97) & $87(73-100)$ & 0.85 & $88(78-98)$ & $70(57-87)$ & 0.006 \\
\hline CR10 after 6MWT & $1.0(0.5-3.0)$ & $1.0(0.2-2.5)$ & $2.0(0.5-3.0)$ & 0.04 & $1.5(0.5-3.0)$ & $2.0(0.5-3.0)$ & 0.94 & $2.0(0.5-3.0)$ & $2.0(0.5-4.0)$ & 0.60 \\
\hline RPE after 6MWT & $11(8-12)$ & $9(8-11)$ & $11(8-13)$ & 0.13 & $11(8-13)$ & $10(8-13)$ & 0.58 & $11(8-13)$ & $9(8-13)$ & 0.62 \\
\hline$S_{\mathrm{pO}_{2}} \min$ & 93 (91-95) & $94(92-96)$ & $92(90-94)$ & $<0.001$ & $93(91-94)$ & 91 (89-94) & 0.004 & 93 (91-94) & $91(90-93)$ & 0.002 \\
\hline $\mathrm{HR}_{\max }$ & $107(99-119)$ & $109(100-122)$ & $107(99-116)$ & 0.17 & $107(100-117)$ & $104(97-113)$ & 0.08 & $107(100-116)$ & $104(96-115)$ & 0.45 \\
\hline MVV \% & $102(89-118)$ & $103(92-119)$ & $100(8-118)$ & 0.12 & $98(87-117)$ & $106(83-119)$ & 0.69 & 101 (89-119) & $116(105-125)$ & 0.11 \\
\hline MIP \% & $58(41-79)$ & $52(37-76)$ & $64(43-82)$ & 0.02 & $61(42-79)$ & $67(49-85)$ & 0.18 & $60(41-79)$ & $68(54-80)$ & 0.26 \\
\hline MEP \% & $79(61-101)$ & $82(65-102)$ & $75(57-100)$ & 0.16 & $76(58-104)$ & $73(56-94)$ & 0.23 & $76(58-101)$ & $64(54-84)$ & 0.08 \\
\hline$P_{0.1} \%$ & $85(58-116)$ & $89(65-125)$ & $80(56-112)$ & 0.02 & $83(57-112)$ & 73 (54-105) & 0.41 & 80 (57-114) & $95(84-172)$ & 0.20 \\
\hline$P_{0.1} /$ MIP ratio & $1.5(0.9-2.6)$ & $1.8(1.0-3.2)$ & $1.4(0.8-2.3)$ & 0.005 & $1.5(0.8-2.4)$ & $1.1(0.8-2.0)$ & 0.11 & $1.4(0.8-2.6)$ & $0.9(0.8-1.7)$ & 0.12 \\
\hline$D_{\mathrm{Lco}} / V_{\mathrm{A}} \%$ & $101(89-112)$ & $101(91-113)$ & $101(86-111)$ & 0.10 & $101(89-111)$ & $102(80-114)$ & 0.85 & $101(88-111)$ & $90(71-107)$ & 0.05 \\
\hline$R_{\mathrm{aw}} \%$ & $140(117-173)$ & $147(120-177)$ & $136(114-168)$ & 0.03 & $143(121-137)$ & $122(102-147)$ & $<0.001$ & $142(120-182)$ & $117(92-147)$ & 0.007 \\
\hline RV \% & $123(105-140)$ & $133(117-151)$ & $116(97-133)$ & $<0.001$ & $121(108-137)$ & $99(88-113)$ & $<0.001$ & $122(107-135)$ & $92(77-109)$ & $<0.001$ \\
\hline TLC \% & $109(98-117)$ & $111(103-121)$ & 106 (95-114) & $<0.001$ & $110(101-116)$ & $97(84-107)$ & $<0.001$ & $109(98-116)$ & $85(82-101)$ & $<0.001$ \\
\hline RV/TLC \% & $39(35-45)$ & $39(36-44)$ & $39(35-45)$ & 0.80 & $40(35-45)$ & $37(34-43)$ & 0.05 & $40(36-46)$ & $38(32-44)$ & 0.05 \\
\hline $\mathrm{FEV}_{1} \%$ & $107(96-117)$ & $107(96-116)$ & $106(96-117)$ & 0.93 & $107(98-117)$ & $105(89-117)$ & 0.29 & $107(97-117)$ & $96(81-116)$ & 0.07 \\
\hline FVC $\%$ & $106(95-117)$ & $107(97-116)$ & 105 (94-116) & 0.24 & $108(97-116)$ & $97(86-112)$ & 0.004 & $106(96-116)$ & $91(80-111)$ & 0.004 \\
\hline $\mathrm{FEV}_{1} / \mathrm{FVC} \%$ & $82(80-86)$ & $82(79-86)$ & $82(78-86)$ & 0.77 & $82(79-86)$ & $83(78-88)$ & 0.45 & $82(78-87)$ & $86(81-88)$ & 0.07 \\
\hline PEF \% & $105(93-118)$ & $102(90-114)$ & $108(94-123)$ & 0.009 & $107(93-121)$ & $109(97-125)$ & 0.23 & $105(93-119)$ & $113(96-126)$ & 0.30 \\
\hline $\mathrm{MEF}_{75 \%}$ & 109 (94-125) & $106(94-118)$ & $112(95-129)$ & 0.02 & $111(94-125)$ & $116(103-131)$ & 0.07 & $109(91-123)$ & $124(107-135)$ & 0.05 \\
\hline $\mathrm{MEF}_{50 \%}$ & $94(76-116)$ & $94(75-115)$ & $95(76-120)$ & 0.72 & $94(75-116)$ & $101(77-128)$ & 0.12 & $93(75-115)$ & $110(87-127)$ & 0.04 \\
\hline $\mathrm{MEF}_{25 \%}$ & $65(48-91)$ & 65 (50-93) & $65(46-86)$ & 0.36 & $66(47-86)$ & $61(44-94)$ & 0.60 & $65(47-89)$ & $72(53-104)$ & 0.31 \\
\hline$F_{\mathrm{ENO}}$ & $13(8-22)$ & $15(10-22)$ & $12(7-24)$ & 0.63 & $15(7-27)$ & $11(6-22)$ & 0.68 & $12(7-27)$ & $9(7-14)$ & 0.72 \\
\hline
\end{tabular}

Data are presented as $\mathrm{n}$, median (interquartile range) or $\mathrm{n}(\%)$, unless otherwise stated. ARDS: acute respiratory distress syndrome; IMV: invasive mechanical ventilation; $\mathrm{S}_{\mathrm{pO}}$ : partial oxygen saturation; SBP: systolic blood pressure; DBP: diastolic blood pressure; CR10: Borg category-ratio scale; RPE: rate of perceived exertion; mMRC: modified Medical Research Council score; 6MWD: 6-min walk distance; $6 \mathrm{MWT}$ : 6-min walk test; $\mathrm{HR}_{\max }$ : maximum heart rate; MVV: maximal voluntary ventilation; MIP: maximal inspiratory pressure; MEP: maximal expiratory pressure; $P_{0.1}$ : airway occlusion pressure after $0.1 \mathrm{~s} ; D_{\mathrm{LCO}}$ : diffusing capacity of the lung for carbon monoxide; $V_{\mathrm{A}}$ : alveolar volume; $R_{\mathrm{aw}}$ : specific airway resistance; RV: residual volume; TLC: total lung capacity; FEV $\mathrm{V}_{1}$ : forced expiratory volume in $1 \mathrm{~s}$; FVC: forced vital capacity; PEF: peak expiratory flow; $\mathrm{MEF}_{75 \%}, \mathrm{MEF}_{50 \%}, \mathrm{MEF}_{25 \%}$ : maximum expiratory flows at $75 \%, 50 \%, 25 \%$, respectively, of forced vital capacity; $F_{\mathrm{ENO}}$ : exhaled nitric oxide fraction. 
TABLE 4 Pearson's correlations with partial oxygen saturation $\left(\mathrm{S}_{\mathrm{pO}_{2}}\right)$ /inspiratory oxygen fraction $\left(F_{\mathrm{IO}_{2}}\right)$ ratio and pneumonia severity index (PSI) during coronavirus disease 2019

\begin{tabular}{|c|c|c|c|c|c|c|}
\hline & \multicolumn{3}{|c|}{$S_{\mathrm{po}_{2}} / F_{\mathrm{IO}_{2}}$ ratio } & \multicolumn{3}{|c|}{ PSI } \\
\hline & Pneumonia & Non-ARDS & ARDS & Pneumonia & Non-ARDS & ARDS \\
\hline Patients n & 222 & 161 & 61 & 222 & 161 & 61 \\
\hline $\mathrm{S}_{\mathrm{pO}_{2}}$ at rest & $0.341^{\star \star}$ & $0.426^{\star \star}$ & $0.302^{*}$ & $-0.376^{\star \star}$ & $-0.351^{\star \star}$ & -0.205 \\
\hline Heart rate at rest & -0.063 & 0.102 & -0.159 & -0.104 & -0.187 & -0.135 \\
\hline Respiratory rate & 0.020 & -0.064 & 0.021 & -0.011 & -0.009 & 0.114 \\
\hline SBP & -0.104 & -0.070 & 0.270 & $0.245^{\star}$ & $0.314^{\star \star}$ & 0.113 \\
\hline DBP & -0.113 & 0.094 & 0.027 & 0.084 & 0.065 & 0.030 \\
\hline mMRC & 0.010 & -0.025 & $0.344^{*}$ & -0.026 & -0.071 & 0.046 \\
\hline$S_{\mathrm{pO}_{2}}$ during $6 \mathrm{MWT}$ & $0.345^{\star *}$ & $0.422^{\star *}$ & 0.329 & $-0.373^{\star \star}$ & $-0.363^{\star \star}$ & -0.150 \\
\hline Heart rate during $6 \mathrm{MWT}$ & 0.140 & 0.148 & 0.178 & $-0.368^{\star \star}$ & $-0.371^{\star \star}$ & $-0.416^{\star}$ \\
\hline 6MWD \% & 0.104 & -0.014 & $0.396^{*}$ & 0.032 & 0.099 & -0.043 \\
\hline MVV & -0.023 & 0.084 & $-0.353^{\star}$ & -0.041 & -0.136 & -0.065 \\
\hline MIP \% & 0.012 & 0.108 & 0.096 & 0.157 & $0.228^{\star}$ & -0.195 \\
\hline MEP \% & $0.208^{*}$ & 0.156 & 0.135 & -0.144 & 0.029 & $-0.389^{*}$ \\
\hline$P_{0.1} \%$ & 0.130 & 0.035 & 0.235 & 0.012 & 0.119 & 0.001 \\
\hline$P_{0.1} / \mathrm{MIP}$ & 0.091 & -0.009 & $0.321^{\star}$ & -0.125 & -0.044 & 0.070 \\
\hline$D_{\mathrm{Lco}} / V_{\mathrm{A}}$ & 0.112 & -0.055 & $0.352^{*}$ & 0.016 & 0.119 & -0.021 \\
\hline$R_{\text {aw }} \%$ & 0.115 & 0.038 & 0.104 & -0.141 & -0.204 & 0.056 \\
\hline RV \% & $0.394^{\star \star}$ & $0.193^{\star}$ & 0.093 & $-0.376^{\star}$ & $-0.248^{\star}$ & -0.077 \\
\hline TLC \% & $0.376^{\star \star}$ & $0.199^{\star}$ & 0.271 & $-0.276^{\star}$ & -0.092 & -0.225 \\
\hline $\mathrm{RV} / \mathrm{TLC}$ & 0.125 & -0.031 & 0.049 & 0.044 & 0.100 & 0.333 \\
\hline $\mathrm{FEV}_{1} \%$ & 0.083 & 0.080 & 0.193 & -0.034 & 0.077 & -0.087 \\
\hline FVC $\%$ & $0.238^{\star \star}$ & $0.198^{*}$ & 0.266 & $-0.198^{\star}$ & -0.060 & -0.143 \\
\hline $\mathrm{FEV}_{1} / \mathrm{FVC}$ & -0.119 & -0.116 & -0.032 & -0.058 & -0.020 & $-0.410^{*}$ \\
\hline PEF \% & -0.061 & 0.103 & 0.041 & 0.047 & 0.092 & -0.179 \\
\hline \multicolumn{7}{|c|}{$\begin{array}{l}\text { ARDS: acute respiratory distress syndrome; SBP: systolic blood pressure; DBP: diastolic blood pressure; mMRC: } \\
\text { modified Medical Research Council score, } 6 \text { MWT: } 6 \text {-min walk test; } 6 \mathrm{MWD}: 6 \text {-min walk distance; MVV: maximal } \\
\text { voluntary ventilation; MIP: maximal inspiratory pressure; MEP: maximal expiratory pressure; } P_{0.1}: \text { airway } \\
\text { occlusion pressure after } 0.1 \mathrm{~s} ; D_{\mathrm{Lco}} \text { : diffusing capacity of the lung for carbon monoxide; } V_{\mathrm{A}} \text { : alveolar volume; } \\
R_{\text {aw }} \text { : specific airway resistance; RV: residual volume; TLC: total lung capacity; FEV } \mathrm{V}_{1} \text { : forced expiratory volume in } \\
1 \mathrm{~s} \text {; FVC: forced vital capacity; PEF: peak expiratory flow. }{ }^{*}: p<0.05 ;{ }^{* \star}: p<0.01 .\end{array}$} \\
\hline
\end{tabular}

$(p=0.01, \beta=0.170)$ and inversely correlated with RV $(p=0.02, \beta=-0.181)$, TLC $(p=0.01, \beta=-0.197), F_{1}$ $(p=0.01, \beta=-0.226)$ and FVC $(p=0.02, \beta=-0.211)$.

\section{Time-dependent parameters}

Evaluating all parameters by time after diagnosis, figure 2 shows the direct correlation between days from diagnosis and $D_{\mathrm{LCO}} / V_{\mathrm{A}}(\mathrm{p}<0.001)$ and 6MWD $(\mathrm{p}=0.004)$.

Health-related quality of life and IPAQ and their correlation with lung function parameters Median (IQR) PCS was 45.8 (37.9-50.9) and MCS was 50.9 (40.9-57.2). IPAQ showed a good prevalence of active patients: $81.7 \%$ of subjects declared an adequate amount of physical activity.

There was no significant correlation between PCS, MCS or IPAQ and lung function, development of pneumonia, ARDS, IMV, $S_{\mathrm{pO}_{2}} / F_{\mathrm{IO}_{2}}$ ratio or PSI.

\section{Discussion}

From previous experience with coronavirus lung involvement of SARS and MERS, radiological abnormalities, impairment of pulmonary function and reduced exercise capacity improve over time, but may be persistent in some for months or even years [1-3].

Evidence about pulmonary function tests after discharge among COVID-19 patients is currently limited to a few retrospective studies with small samples: a reduction of FVC 6 weeks after discharge in 13 patients [4]; reduction of $D_{\mathrm{LCO}}$, TLC and 6MWD in severe COVID-19 compared to non-severe COVID-19 at 30 days, and impairment in respiratory muscle strength in more than half of the subjects [5]; and reduction 

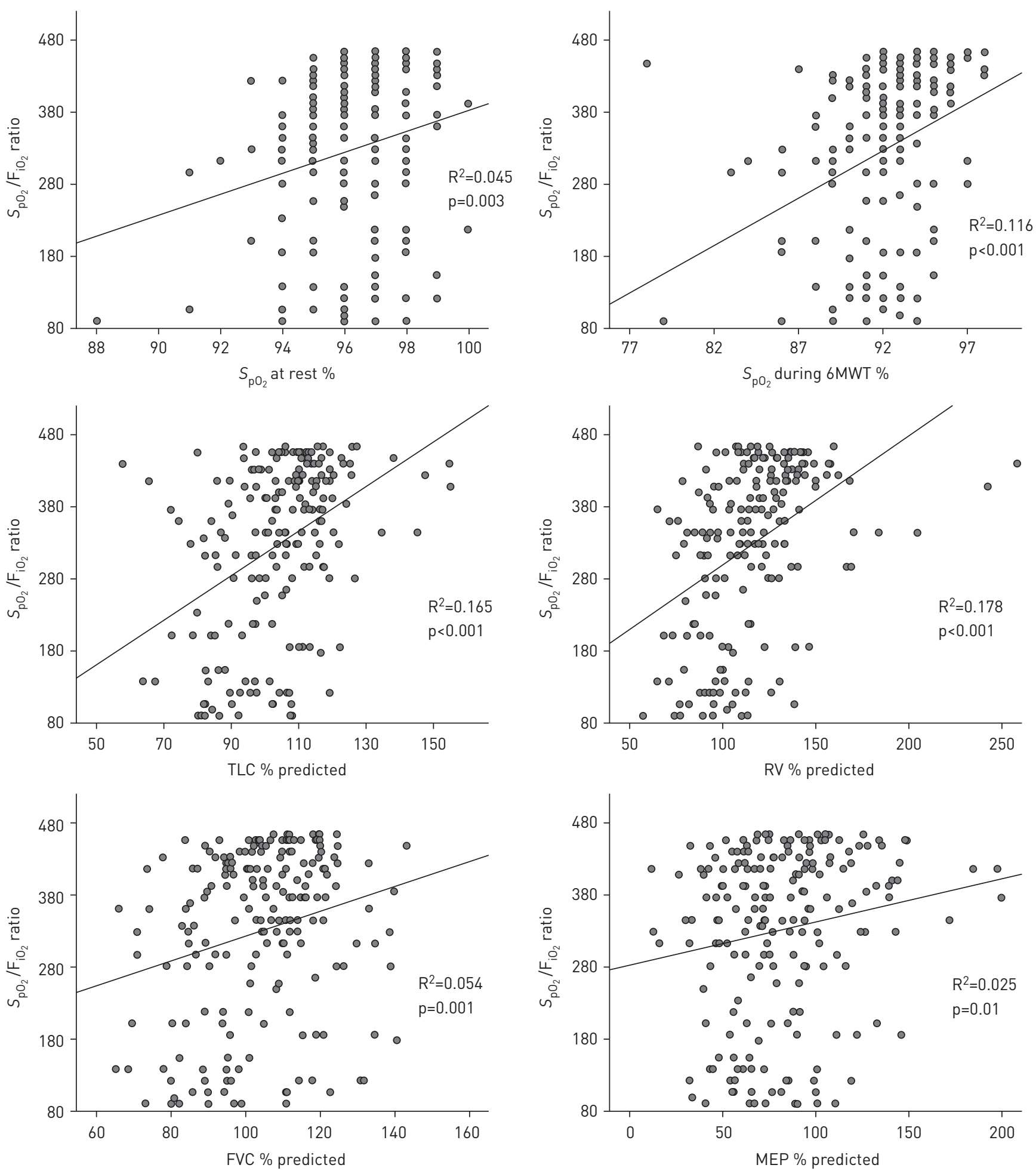

FIGURE 1 Scatter plot showing significant correlation between partial oxygen saturation $\left(\mathrm{S}_{\mathrm{pO}_{2}}\right) /$ inspiratory oxygen fraction $\left(F_{1 \mathrm{O}_{2}}\right)$ ratio during severe acute respiratory syndrome coronavirus 2 disease and pulmonary function and 6-min walk test (6MWT) parameters. TLC: total lung capacity; RV: residual volume; FVC: forced vital capacity; MEP: maximal expiratory pressure.

of TLC, $\mathrm{FEV}_{1}$, FVC, $D_{\mathrm{LCO}}$ and small airway function at 3 months in a small number of patients [7] have been reported. In all studies, a reduction of $D_{\mathrm{LCO}}$ but not $D_{\mathrm{LCO}} / V_{\mathrm{A}}$ was noted; this finding was related to the reduction of $V_{\mathrm{A}}$ without residual interstitial abnormalities or pulmonary vascular abnormalities [14]. 
a)

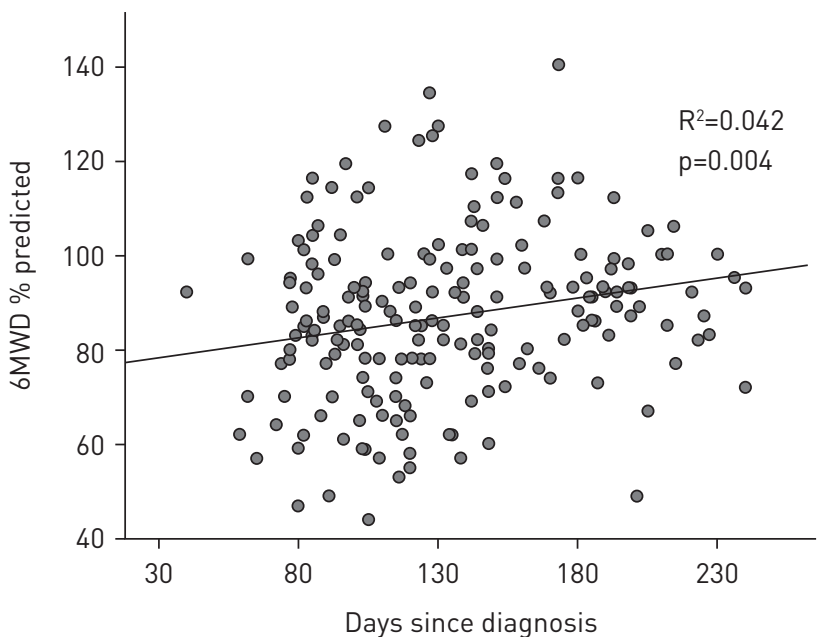

b)

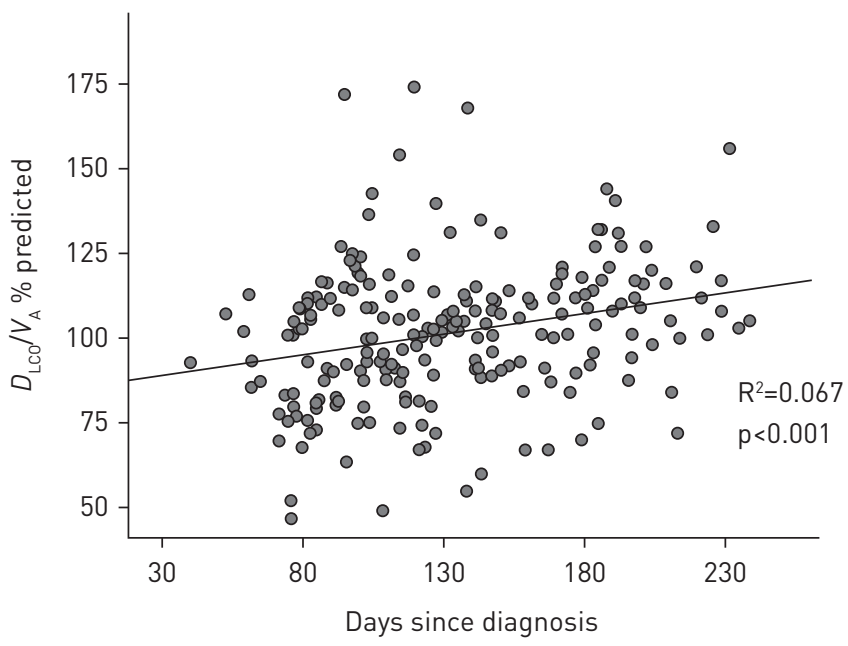

FIGURE 2 Scatter plot showing significant correlation between time since diagnosis and a) 6-min walk distance (6MWD) and b) diffusing capacity of the lung for carbon monoxide $\left(D_{\mathrm{LCO}}\right) /$ alveolar volume $\left(V_{\mathrm{A}}\right)$ ratio.

The proposed pathogenic mechanism assumes initial damage induced by COVID-19, similar to that induced by SARS, due to microvascular injury with initial interstitial thickening with clear lungs on radiology exams along with a profound hypoxaemia [15, 16], followed by the development of alveolar damage inducing a gradual loss of the alveolar spaces [16]. The decreased $V_{\mathrm{A}}$ may be explained by temporary changes in mechanical properties of the chest wall and respiratory muscles after critical illness and are supposed to encompass a possible long-lasting pulmonary parenchymal dysfunction post-COVID-19 [17].

To our knowledge, our study is the first with a large sample on this subject. Our results show a reduction of respiratory function and exercise capacity secondary to SARS-CoV-2 pneumonia, mostly in patients who developed ARDS during the acute phase. The severity of pneumonia, assessed by the development of ARDS, need of IMV, worst $S_{\mathrm{pO}_{2}} / F_{\mathrm{IO}_{2}}$ ratio and PSI during acute COVID-19 seems to be associated with the reduction of $D_{\mathrm{LCO}} / V_{\mathrm{A}}$ ratio, and secondarily with the reduction of $S_{\mathrm{pO}_{2}}$ at rest and during the 6MWT, but also impacting on 6MWD, heart rate during 6MWT, MEP, TLC, RV and FVC. Effect on $D_{\mathrm{LCO}} / V_{\mathrm{A}}$ and 6MWD appear to decrease over time. Interestingly, patients without pneumonia compared to patients with pneumonia during COVID-19 showed greater increases in $R_{\mathrm{aw}}$ and RV, but comparable RV/TLC ratios, describing a possible involvement of lower airways instead of lung parenchyma. Respiratory muscle weakness, as reflected by decreased MIP and MEP, could be due to different factors, such as a myopathy caused by the virus in respiratory muscles, especially in the diaphragm, or could be a possible effect of limited physical activity secondary to the lockdown. In IMV patients, decreased MEP could be explained by the combination of curarisation, corticosteroids and lack of spontaneous respiratory movements for several days. $P_{0.1}$ and $P_{0.1}$ MIP were significantly decreased in pneumonia patients, describing a possible neural-drive impairment in these patients. These results appear to be independent of the different prevalence of obesity in the two groups.

The correlation between steroid treatment and dyspnoea scale and some respiratory functional values appears to be independent of pneumonia severity and could reflect the contribution of steroids to viral myopathy through several mechanisms, such as altered electrical excitability of muscle fibres, loss of thick filaments and inhibition of protein synthesis [18-20], even if MIP and MEP did not prove this relationship.

Evaluating health-related quality of life, a reduction in physical health was found; however, normal mental health was reported despite the long period of isolation and extreme uncertainty during COVID-19, which could have created psychological and mood disturbances.

The reduction of respiratory function and exercise capacity observed after COVID-19 were more pronounced in patients who developed ARDS or required IMV. In these patients, in order to obtain a rapid restoration of normal functional parameters, respiratory rehabilitation and gradual physical activity seem to 
be effective tools, as shown in a randomised trial of 6-week respiratory rehabilitation after discharge, which showed improvement in respiratory function $\left(\mathrm{FEV}_{1}, \mathrm{TLC}, \mathrm{FEV}_{1} / \mathrm{FVC}, 6 \mathrm{MWD}\right.$ and $D_{\mathrm{LCO}}$ ), quality of life and anxiety in older patients [6].

According to these results, respiratory evaluation does not appear to be necessary in patients without pneumonia and without symptoms. $D_{\text {LCO }}, 6 \mathrm{MWT}$ and plethysmography could be avoided in patients without pneumonia, performing only spirometry with bronchodilator responsiveness testing and recommending early resumption of physical activity. In contrast, in patients who developed ARDS, $D_{\text {LCO, }}$ 6MWT and complete spirometry could uncover presence of residual pulmonary and functional impairment, with need for respiratory rehabilitation and gradual physical activity.

There are several limitations to this study: firstly, the lack of pulmonary function tests before COVID-19 infection; secondly, we assessed inspiratory and expiratory muscle strength with mouth pressure, but low values might result from technical difficulties (the functional disability appears to be out of proportion to the degree of lung function impairment and may be due to additional factors such as muscle deconditioning and steroid myopathy); and lastly, the lack of correlation between pulmonary function and radiological signs.

Our study will provide another prospective evaluation after 12 months to better understand the respiratory long-term sequelae of SARS-CoV-2 disease. Long-term follow-up studies are needed to better understand the impact of SARS-CoV-2 on human pathophysiology.

\section{Conclusion}

Patients with severe lung involvement during SARS-CoV-2 infection showed impaired pulmonary function test parameters and 6MWT $S_{\mathrm{pO}_{2}}$ values 4 months after the acute illness. Clinical and instrumental long-term checks on these patients are advisable, enabling a respiratory rehabilitation course aimed at respiratory recovery.

Author contributions: F. Anastasio, S. Barbuto and E. Scarnecchia contributed equally to the writing of the manuscript.

Conflict of interest: None declared.

\section{References}

1 Das KM, Lee EY, Singh R, et al. Follow-up chest radiographic findings in patients with MERS-CoV after recovery. Indian J Radiol Imaging 2017; 27: 342-349.

2 Hui DS, Joynt GM, Wong KT, et al. Impact of severe acute respiratory syndrome (SARS) on pulmonary function, functional capacity and quality of life in a cohort of survivors. Thorax 2005; 60: 401-409.

3 Zhang P, Li J, Liu H, et al. Long-term bone and lung consequences associated with hospital-acquired severe acute respiratory syndrome: a 15-year follow-up from a prospective cohort study. Bone Res 2020; 8: 8.

4 Fumagalli A, Misuraca C, Bianchi A, et al. Pulmonary function in patients surviving to CoVID-19 pneumonia. Infection 2021; 49: 153-157.

5 Huang Y, Tan C, Wu J, et al. Impact of coronavirus disease 2019 on pulmonary function in early convalescence phase. Respir Res 2020; 21: 163.

6 Liu K, Zhang W, Yang Y, et al. Respiratory rehabilitation in elderly patients with COVID-19: a randomized controlled study. Complement Ther Clin Pract 2020; 39: 101166.

7 Zhao $\mathrm{Y}$, Shang $\mathrm{Y}$, Song $\mathrm{W}$, et al. Follow-up study of the pulmonary function and related physiological characteristics of COVID-19 survivors three months after recovery. EClinicalMedicine 2020; 25: 100463.

8 The ARDS Definition Task Force. Acute respiratory distress syndrome: the Berlin definition. JAMA 2012; 307: 2526-2533.

9 Tyrrell CSB, Mytton OT, Gentry SV, et al. Managing intensive care admissions when there are not enough beds during the COVID-19 pandemic: a systematic review. Thorax 2021; 76: 302-312.

10 Quanjer PH, Stanojevic S, Cole TJ, et al. Multi-ethnic reference values for spirometry for the 3-95-yr age range: the global lung function 2012 equations. Eur Respir J 2012; 40: 1324-1343.

11 ATS Committee on Proficiency Standards for Clinical Pulmonary Function Laboratories. ATS statement: guidelines for the six-minute walk test. Am J Respir Crit Care Med 2002; 166: 111-117.

12 Ware J Jr, Kosinski M, Keller S. SF-12: How to Score the SF-12 Physical and Mental Health Summary Scales. Boston, Health Institute, New England Medical Center, 1998. 
13 Van Dyck D, Cardon G, Deforche B, et al. IPAQ interview version: convergent validity with accelerometers and comparison of physical activity and sedentary time levels with the self-administered version. $J$ Sports Med Phys Fitness 2015; 55: 776-786.

14 Nusair S. Abnormal carbon monoxide diffusion capacity in COVID-19 patients at time of hospital discharge. Eur Respir J 2020; 56: 2001832.

15 McGonagle D, O'Donnell JS, Sharif K, et al. Immune mechanisms of pulmonary intravascular coagulopathy in COVID-19 pneumonia. Lancet Rheumatol 2020; 2: e437-e445.

16 Tian S, Hu W, Niu L, et al. Pulmonary pathology of early-phase 2019 novel coronavirus (COVID-19) pneumonia in two patients with lung cancer. J Thorac Oncol 2020; 15: 700-704.

17 Spagnolo P, Balestro E, Aliberti S, et al. Pulmonary fibrosis secondary to COVID-19: a call to arms? Lancet Respir Med 2020; 8: 750-752.

18 Dekhuijzen PN, Decramer M. Steroid-induced myopathy and its significance to respiratory disease: a known disease rediscovered. Eur Respir J 1992; 5: 997-1003.

19 Nava S, Fracchia C, Callegari G, et al. Weakness of respiratory and skeletal muscles after a short course of steroids in patients with acute lung rejection. Eur Respir J 2002; 20: 497-499.

20 Polsonetti BW, Joy SD, Laos LF. Steroid-induced myopathy in the ICU. Ann Pharmacother 2002; 36: 1741-1744. 